\title{
PRE-LAUNCH ALGORITHM AND DATA FORMAT FOR THE LEVEL 1 CALIBRATION PRODUCTS FOR THE EOS AM-1 MODERATE RESOLUTION IMAGING SPECTRORADIOMETER (MODIS)
}

B. Guenther, Gerald D. Godden, Xiaoxiong Xiong, Edward J. Knight, Shi-Yue Qiu Harry Montgomery, M. M. Hopkins, Mohammad G. Khayat and Zhidong Hao

\author{
Submitted to Transactions on Geoscience and Remote Sensing \\ EOS-AM Special Issue
}

April 17, 1998

ABSTRACT---The MODIS radiometric calibration product (Level 1B) is described for the thermal emissive and the reflective solar bands. Specific sensor design characteristics are identified to assist in understanding how the calibration algorithm software product is designed. The reflected solar band software products of radiance and reflectance factor both are described. The product file format is summarized and the MCST Homepage location for the current file format is provided.

Guenther and Montgomery are employed by NASA's Goddard Space Flight Center, Greenbelt, MD 20771. Godden is employed by Physics Application, Inc, Vienna, VA 22182

Xiong is employed by Science Systems and Applications, Inc, Lanham, MD 20706

Knight and Khayat are employed by Research and Data Systems Corporation, Greenbelt, MD 20770

Qiu is employed by Computation Physics Applications, Inc. Columbia MD 21042

Hopkins and Hao are employed by General Sciences Corporation, Laurel, MD 20707

Corresponding Author: B. Guenther, Mail Code 920.1, GSFC, Greenbelt, MD 20771; telephone (301)2865205; fax (301)286-0373; email: guenther@ltpmail.gsfc.nasa.gov 


\subsection{Introduction}

This paper describes the MODIS calibration data product. The calibration equations for this product are developed and the approaches for the laboratory calibration and the on-orbit verification of that calibration are described also. The development of the MODIS science products begins with the Level $1 \mathrm{~B}$ calibration products. The radiometric characteristics of the L1B product are reviewed, but the geometric registration and spectral characterization of the products are not described in this paper. The primary aspects of the MODIS sensor is discussed by Barnes [1]. Major science products for the MODIS oceans [2] and land surface [3] research communities are described elsewhere in this issue.

Sensor design and characteristics necessary to understand the Level 1B software product are reviewed in the Section 2.0, Instrument Background. The emissive infrared algorithms are described in Section 3.0. The reflected solar bands algorithm, including subsections on the radiance and the reflectance factor products, is covered in Section 4.0. The Level 1B data software product attributes such as file format and uncertainty index are reviewed in Section 5.0. The conclusion and summary comments are provided in Section 6.0.

\subsection{Instrument Background}

The MODIS is a 36-band spectroradiometer which covers a broad spectral range, and has very demanding calibration performance characteristics. The sensor design incorporates a paddle-wheel scan mirror to provide a wide swath across the Earth surface, and to provide access to an array of characterization sub-systems on each rotation of the mirror. The spectral separation of the light comes from three dichroics and sliver interference filters at each detector. The spectral ranges for the reflected solar bands $(0.4$ to $2.3 \mu \mathrm{m})$ and the emissive infrared bands ( 3.6 to $14.4 \mu \mathrm{m}$ ) calibrated and analyzed with separate techniques. The descriptions of the calibration approach and algorithm follows this separation.

Figure 1 provides a schematic of the sensor optics, optical detectors and electronics to demonstrate how optical signals onto the scan mirror are transformed to digital signals or data. The MODIS design incorporates several on-board calibration (OBC) targets and each is valuable for some aspect of the calibration algorithm design. The scan mirror points to the internal OBCs: the solar diffuser (SD), the spectroradiometric calibration assembly (SRCA), the blackbody (BB) and cold space view (SV) on each mirror rotation. The scan mirror is double sided and both sides are used for the MODIS observations. A rotation of 360 degrees of the scan mirror requires $2.954 \mathrm{sec}$. Throughout this paper, a period of $1.477 \mathrm{sec}$ is identified as a scan, and the data produced from it is called a scan line of data, 
or a scan line. Scan lines alternate between mirror side A (MS A) and mirror side B (MS B) in the data set.

The location of a target within a scan line can be identified by its Principal Scan Angle. The position of each $\mathrm{OBC}$, the location of the Earth view around the scan, and the location of the laboratory calibration sources are shown in Figure 2. The Principal Scan Angle is referenced to NADIR, with a value of zero. Figure 2 also indicates the angle of incidence (AOI) on the scan mirror for each of these targets.

The Earth scenes are sampled 1354 times over a range of principal scan angles of -55 degrees to +55 degrees. All the samples are co-registered in the sensor data system as frames and telemetered together to the ground as frames. At the NADIR a frame is 10,000 $\mathrm{m}$ extent in the track direction (spacecraft orbital direction) and 1,000 $\mathrm{m}$ extent in the scan direction (orthogonal to the spacecraft direction orbital direction). The sensor has bands with three ground resolutions of $250 \mathrm{~m}, 500 \mathrm{~m}$ and $1,000 \mathrm{~m}$. The $1,000 \mathrm{~m}$ bands are sampled once each frame, and are composed of 10 channels (individual detectors) in each band. The $500 \mathrm{~m}$ bands are sampled twice each frame and are composed of 20 channels in each band. The $250 \mathrm{~m}$ bands are sampled four times each frame and are composed of 40 channels in each band. The channels are positioned end-to-end in the track direction for each band on each focal plane.

The MODIS bands are positioned in four separate focal planes, with bands with center wavelengths between 0.42 to $0.55 \mu \mathrm{m}$ on the visible wavelength focal plane (VIS), with center wavelengths 0.64 to $0.94 \mu \mathrm{m}$ on the near-infrared wavelength focal plane (NIR), with center wavelengths 1.2 to $4.5 \mu \mathrm{m}$ on the short-infrared and middle-infrared wavelength focal plane (SW/MWIR) and with center wavelengths 6.5 to $14.2 \mu \mathrm{m}$ on the long-infrared wavelength focal plane (LWIR). The SW/MWIR and LWIR focal planes are housed together within the MODIS radiative cooler. Information on the spectral bandpasses in the sensor specification for the MODIS are available in [1], and the definitive relative spectral responses available through the MCST Homepage on the World Wide Web at http://tpwww.gsfc.nasa.gov/MODIS/MCST/Home.html, under the button "Individual Bands Data."

The important characteristics of the OBC follow:

\subsection{Solar Diffuser}


The solar diffuser is a flat surface. The composition of the surface isSpectralon ${ }^{\mathrm{TM}}$, and the manufacturer specifications of the reflectance of this surface [4] are presented in Figure 3. The reflectance of the this diffuser has very small variations with wavelength across all MODIS reflected solar bands, except for the variation across Band 7.

\subsection{Spectroradiometric Calibration Assembly}

The SRCA is comprised of a source assembly, a monochromator, transfer optics and collimating optics on the output. It includes internal diode detectors to track sub-system characteristics. The SRCA is used on-orbit for spectral bandpass knowledge of the reflected solar bands, and for within an orbit radiometric stability verification of the reflected solar bands. The radiometric sensitivity to temperature variations is determined in pre-launch thermal vacuum testing. The temperature sensitivity is verified on-orbit by tracking the response of MODIS to the SRCA through the intraorbit temperature range, when the SRCA is operated in the radiometric mode.

\subsection{Blackbody}

The BB is of a V-groove design which has an emissivity of 0.993 in the direction it is used by MODIS. 12 thermistors are distributed across the surface of the BB.

\subsection{Space View Port}

The SV is an aperture in the side of MODIS that provides a view of cold space to allow for a zero radiance inpu signal on each scan. This zero input radiance on each scan in the emissive infrared allows a determination of the amount of self radiation. This is important because the sensor operates with "warm" optics which contributes a significant amount of signal for each observation. The signal from a scene will be determined using a subtraction of the sensor self radiation effect from the cold space look on each scan.

\subsection{MODIS Thermal Emissive Bands Radiometric Calibration Algorithm}

The MCST has pursued two independent calibration methodologies for the MODIS thermal bands. The first method is based on the traditional technique of fitting the instrument output digital numbers (DNs) versus radiance $(\mathrm{L})\left[\mathrm{Watts} /\left(\mathrm{m}^{2} * \mathrm{sr} * \mathrm{~m}\right)\right]$ distribution to a quadratic polynomial expression, and is similar in form to the algorithm suggested by Young [5]. This is referred to as the L vs DN algorithm. The second method is based on a somewhat more physical approach of converting the output DNs to detector output voltages (V), incorporating the telemetered detector response to scenes onto the scan mirror with zero-radiance, and fitting these results to a second order equation that applies a local curvature as a function of the signal level. The signal level of the detectors in the second 
method is due to the combination of scene radiance (on to the scan mirror) plus sensor selfgenerated radiance. This is referred to as the $\mathrm{V}$ vs $\mathrm{L}$ algorithm and is described by Knowles [6]. This paper presents an overview of the first method, which is planned to be the baseline algorithm at launch. Investigation of the more complex (and perhaps more physical) V vs $\mathrm{L}$ algorithm performance, and comparison with the $\mathrm{L}$ vs $\mathrm{DN}$ algorithm, is a subject of continuing research, and is not presented here. In this discussion we do not consider the combined effects of possible scene polarization and MODIS MWIR and LWIR optical train polarization. The effects of MODIS polarization on unpolarized scenes are estimated to be small compared to specified radiance uncertainties [7]. These effects will be addressed in a subsequent publication.

The MODIS thermal emissive bands (Bands 20-25, and 27-36) covering the wavelength region from 3.75 to $14.24 \mu \mathrm{m}$, and consisting of 10 channels per band, are radiometrically calibrated on the ground using a precision Blackbody Calibration Source (BCS) which is contained within the Thermal Vacuum chamber with the MODIS during calibration. The BCS is of the "buried-first bounce" type design and is expected to have an emissivity of 0.9995. It is located at $-45^{\circ}$ scan angle with respect to the MODIS NADIR viewing scan position in the $110^{\circ}$ Earth view scan angle range. This translates into a low Angle-ofIncidence (AOI) $\left(15.5^{\circ}\right)$ on the scan mirror to minimize reflectance variation effects. The on-board calibrator blackbody (OBC BB) and the Space View Port are viewed once per scan mirror rotation by each side of the scan mirror. During Thermal Vacuum calibration the space view port is covered with a cryogenically cooled Space View Source (SVS) to simulate the on-orbit view of cold space.

The BCS emissivity is calculated, based on the design of the BCS and a measurement of the reflectance of the materials which comprise the BCS.. The BSC temperature is measured with thermistors which have a temperature calibration traceable to an NIST standard temperature mesurement. Consequently, the MODIS thermal emissive band calibration pedigree is based on a temperature standard, not a radiometric standard.

The silver coated scan mirror exhibits a significant variation in average reflectance as a function of wavelength and angle of incidence of the light, Cafferty, private communication [8] and MacDonald [9] within this spectral region. In addition to the wavelength dependent reflectivity for the scan mirror, the AOI locations of the BCS, OBC $\mathrm{BB}$ and the SVS are critical factors which must be incorporated into the algorithm. The location of these sources along a scan are depicted in Figure 2. 
The pre-launch calibration is based on the laboratory BCS. The BCS calibration is imprinted onto the $\mathrm{OBC} B \mathrm{~B}$, and the on-orbit calibration is maintained with the $\mathrm{OBC} B \mathrm{~B}$ and SV combination.

\subsection{Pre-launch Radiometric Calibration Algorithm}

The MODIS is a conventional differencing radiometer. Instrument background radiation effects are removed by subtracting the cold space view signal from the Earth view signal on a scan-by-scan basis. During Thermal Vacuum calibration the spectral radiance from the Blackbody Calibration Source (BCS), after reflection from the scan mirror is given by:

$$
L_{B C S_{-} P A T H}=r_{B C S}^{s m} e_{B C S} L\left(T_{B C S}\right)+\left(1-r_{B C S}^{s m}\right) L\left(T_{s m}\right)+L_{B K G}{ }^{\prime}
$$

where $\rho_{\mathrm{BCS}}^{\mathrm{sm}}$ is the scan mirror reflectivity integrated over the relative spectral response at the AOI for the BCS, $\varepsilon_{\mathrm{BCs}}$ represents the $\mathrm{BCS}$ emissivity, $\mathrm{L}\left(\mathrm{T}_{\mathrm{BCS}}\right)$ is the radiance calculated from the Planck equation at the BCS temperature $T_{B C S}, L\left(T_{s m}\right)$ is the radiance calculated from the Planck equation at scan mirror temperature $T_{s m}$, and $L_{B K G}$ is the instrument background radiance exclusive of the scan mirror emission. The second term of this equation represents the emission from the scan mirror. This term is separated from the total instrument background to explicitly capture its scan angle and temperature dependence.

Similarly, when the MODIS views the Space View Source, the spectral radiance after the scan mirror is given by:

$$
L_{S V S_{-} P A T H}=\left(1-r_{S V S}^{s m}\right) L\left(T_{s m}\right)+L_{B K G}{ }^{\prime}
$$

To remove the variable instrument background effect, the Space View Source term is subtracted from the input signal. Thus, the spectral radiance difference is:

$$
D L_{B C S}=L_{B C S_{-} P A T H}-L_{S V S_{-} P A T H}=r_{B C S}^{s m} e_{B C S} L\left(T_{B C S}\right)+\left(r_{S T S}^{s m}-r_{B C S}^{s m}\right) L\left(T_{s m}\right)
$$

For a specific MODIS thermal emissive band (B), the band averaged radiance difference due the BCS path and the SVS path is:

$$
D L_{B C S}(B)=r_{B C S}^{s m} e_{B C S} \overline{L\left(T_{B C S}\right)}+\left(r_{S V S}^{s m}-r_{B C S}^{s m} \overline{L\left(T_{s m}\right)}\right.
$$

where 


$$
\left.\overline{L\left(T_{B C S}\right.}\right)=\frac{\int L\left(T_{B C S}, l\right) R S R(B, l) d l}{\int R S R(B, l) d l}
$$

and $\operatorname{RSR}(B, \lambda)$ represents the wavelength dependent Relative Spectral Response (normalized to unity at peak) for band $B$, and a similar expression for the band integration applies to the second term on the right hand side of Eqn. 4. N.B., through out this discussion, the channel number indices are suppressed for clarity.

The band integrated radiance difference $\Delta \mathrm{L}_{\mathrm{BCS}}(\mathrm{B})$ is a function of $\mathrm{DN}_{\mathrm{BCS}}-\mathrm{DN}_{\mathrm{SVS}}$. Let

$$
d n_{B C S}=\frac{1}{N_{s c a n s}} \sum_{n=1}^{N_{x a n s}}\left[\frac{1}{M_{B C S}} \sum_{m=1}^{M_{B C s}} D N_{B C S}-\frac{1}{M_{S V S}} \sum_{m=1}^{M_{S v s}} D N_{S V S}\right]
$$

where $\mathrm{M}_{\mathrm{BCS}}$ (\# of BCS frames), $\mathrm{M}_{\mathrm{svs}}$ (\# of SVS frames), and $\mathrm{N}_{\mathrm{scans}}$ (\# of scans) are appropriately smoothing intervals to optimize the calibration coefficients.

On the basis of experience with similar instruments, and observed temperature dependencies, we postulate a temperature dependent second order nonlinear behavior for $\Delta \mathrm{L}_{\mathrm{BCS}}\left(\mathrm{B}, \mathrm{T}_{\mathrm{instr}}\right)$

$$
D L_{B C S}\left(B, T_{\text {instr }}\right)=a_{0}^{B C S}\left(B, T_{\text {instr }}\right)+a_{1}^{B C S}\left(B, T_{\text {instr }}\right) \cdot d n_{B C S}+a_{2}^{B C S}\left(B, T_{\text {instr }}\right) \cdot\left(d n_{B C S}\right)^{2}
$$

or,

$$
\begin{aligned}
& r_{B C S}^{s m} e_{B C S} \overline{L\left(T_{B C S}\right)}+\left(r_{S V S}^{s m}-r_{B C S}^{s m}\right) L \overline{L\left(T_{s m}\right)} \\
= & a_{0}^{B C S}\left(B, T_{i n s t r}\right)+a_{1}^{B C S}\left(B, T_{i n s t r}\right) \cdot d n_{B C S}+a_{2}^{B C S}\left(B, T_{i n s t r}\right) \cdot\left(d n_{B C S}\right)^{2}
\end{aligned}
$$

The $B C S$ calibration coefficients $\mathrm{a}_{0}^{\mathrm{BCS}}\left(\mathrm{B}, \mathrm{T}_{\text {instr }}\right), \mathrm{a}_{1}^{\mathrm{BCS}}\left(\mathrm{B}, \mathrm{T}_{\text {inst }}\right)$, and $\mathrm{a}_{2}^{\mathrm{BCS}}\left(\mathrm{B}, \mathrm{T}_{\text {instr }}\right)$ are determined by least-squares fitting to the corresponding data. In this formulation, $\mathrm{a}_{0}^{\mathrm{BCS}}\left(\mathrm{B}, \mathrm{T}_{\mathrm{instr}}\right)$ term is viewed as part of the least squares fitting process. It is expected to take on small non-zero values. The contribution from the nonlinear response term $a_{2}^{B C S}\left(B, T_{\text {instr }}\right)$ is very small compared to the linear response term. Apart from the temperature dependent behavior, both $\mathrm{a}_{0}^{\mathrm{BCS}}\left(\mathrm{B}, \mathrm{T}_{\text {instr }}\right)$ and $\mathrm{a}_{2}^{\mathrm{BCS}}\left(\mathrm{B}, \mathrm{T}_{\text {instr }}\right)$ terms will be fixed for the on-orbit operation. 


\subsection{On-Orbit Radiometric Algorithm}

When the MODIS views the on-board blackbody (OBC BB), the spectral radiance after the scan mirror includes the $\mathrm{OBC} B \mathrm{BB}$ emitted radiance reflected by the scan mirror, the scan mirror emittance, the scan cavity emittance reflected by the $\mathrm{OBC} B \mathrm{BB}$ and then in turn by the scan mirror, and the remaining instrument background radiance. Thus

$$
L_{B B_{-} P A T H}=e_{B B} L\left(T_{B B}\right) r_{B B}^{s m}+\left(1-r_{B B}^{s m}\right) L\left(T_{s m}\right)+e_{c a v} L\left(T_{c a v}\right)\left(1-e_{B B}\right) r_{B B}^{s m}+L_{B K G}^{\prime}
$$

where $\varepsilon_{\mathrm{BB}}$ is the $\mathrm{OBC} \mathrm{BB}$ emissivity, $\left(1-\varepsilon_{\mathrm{BB}}\right)$ is the reflectivity of the $\mathrm{OBC} \mathrm{BB}$, and $\varepsilon_{\text {cav }}$ represents the MODIS scan cavity effective emissivity which must account for the Earth and space view apertures.

From Eqn. 9 and 2, the spectral radiance difference attributed to the $\mathrm{OBC} B \mathrm{BB}$ path and the $\mathrm{s}$ space view path is given by:

$$
\begin{aligned}
\Delta L_{B B} & =L_{B B_{-} P A T H}-L_{S V_{-} A_{A T H}} \\
& =r_{B B}^{s m} e_{B B} L\left(T_{B B}\right)+\left(r_{S V}^{s m}-r_{B B}^{s m}\right) L\left(T_{s m}\right)+\left(1-e_{B B}\right) e_{c a v} r_{B B}^{s m} L\left(T_{c a v}\right)
\end{aligned}
$$

For a specific MODIS band, the band averaged radiance difference between the OBC BB path and the space view path is given by:

$$
D L_{B B}(B)=r_{B B}^{s m} e_{B B} \overline{L\left(T_{B B}\right)}+\left(r_{S V}^{s m}-r_{B B}^{s m}\right) \overline{L\left(T_{s m}\right)}+\left(I-e_{B B}\right) e_{C A V} r_{B B}^{s m} \overline{L\left(T_{C A V}\right)}
$$

where $\overline{\mathrm{L}\left(\mathrm{T}_{\mathrm{BB}}\right)}, \overline{\mathrm{L}\left(\mathrm{T}_{\mathrm{sm}}\right)}$, and $\overline{\mathrm{L}\left(\mathrm{T}_{\mathrm{CAV}}\right)}$ are the Planck emission terms, determined using Eqn. 5 .

The earth view sector radiance is determined using the on-orbit calibration coefficient obtained with the OBC BB and the space view, and the pre-launch second order calibration coefficients determined from the Thermal Vacuum BCS data sets. Eqn. 12 describes the use of these coefficients:

$$
D L_{E V}\left(B, T_{\text {instr }}\right)=a_{0}^{B C S}\left(B, T_{\text {instr }}\right)+b_{1}^{B B}\left(B, T_{\text {instr }}\right) \bullet d n_{E V}+a_{2}^{B C S}\left(B, T_{\text {instr }}\right) \bullet\left(d n_{E V}\right)^{2}
$$

where the residual offset coefficient $\mathrm{a}_{0}^{\mathrm{BCS}}\left(\mathrm{B}, \mathrm{T}_{\text {instr }}\right)$ and second order coefficient, $a_{2}^{B C S}\left(B, T_{\text {instr }}\right)$ are determined using Eqn. 8, and the linear response $b_{1}^{B B}\left(B, T_{i n s t r}\right)$ is determined on a scan-by-scan basis by 


$$
b_{1}^{B B}(B)=\frac{D L_{B B}(B)-a_{0}^{B C S}(B)-a_{2}^{B C S}(B) \bullet\left(d n_{B B}\right)^{2}}{d n_{B B}}
$$

where $\Delta \mathrm{L}_{\mathrm{BB}}(\mathrm{B})$ is given by Eqn. 11, and

$$
d n_{B B}=\frac{1}{M_{B B}} \sum_{i=1}^{M_{B g}} D N_{B B}-\frac{1}{M_{S V S}} \sum_{i=1}^{M_{S V S}} D N_{S V S}
$$

To achieve slowly varying behavior the linear response term is averaged over $\mathrm{N}_{\text {scans }}$

$$
\overline{b_{l}^{B B}(B)}=\frac{I}{N_{\text {Scans }}} \sum_{j=-N_{\text {som }} / 2}^{N_{\text {soou }} / 2} b_{l, j}^{B B}(B)
$$

The MODIS scan mirror takes 2.954 seconds to complete a rotation. During each scan a new measurement of the linear response (gain) is determined, according to Eqn. 14. The drift of the gain from scan to scan is expected to be very small. This slowly varying behavior is considered by averaging the linear response term over $N_{\text {scans }}$ as shown in Eqn. 15.

The OBC BB will be cycled to $315 \mathrm{~K}$ and allowed to return to the sensor thermal ambient temperature on an approximately bi-weekly basis. This $\mathrm{OBC}$ elevated temperature operation will simulate the ground calibration process from which the calibration coefficients were calculated. This cycle will allow for a determination of $b_{1}{ }^{\text {BB-elev }}(B)$. Comparison of $b_{1}{ }^{\text {BB-elev }}(B)$ with $b_{1}{ }^{\mathrm{BB}}\left(\mathrm{B}, \mathrm{T}_{\text {instr }}\right)$ will verify the continued stability of on-orbit operation of $\mathrm{a}_{0}{ }^{\mathrm{BCS}}$ and $\mathrm{a}_{2}{ }^{\mathrm{BCS}}$. Vicarious calibration measurements obtained during the Validation phase of the MODIS also will be used to verify the stability on-orbit operation of $\mathrm{a}_{0}{ }^{\mathrm{BCS}}$ and $\mathrm{a}_{2}{ }^{\mathrm{BCS}}$ and the performance of the OBC BB.

There is a small time difference between when the data from the OBC BB and SV targets are collected for the gain calculation and when the Earth view data is collected. During this time interval the instrument background may be expected to drift a small relative amount due to $1 / \mathrm{f}$ noise. In a manner analogous to that suggested by Knowles [6] a portion of this drift can be reduced by linear interpolation between successive scans.

Using the average gain (i.e. the linear response) the dn difference, $\Delta_{\mathrm{BB}, \mathrm{i}}$, for a given band between the $\mathrm{i}^{\text {th }}$ measurement, and the average of many measurements of the OBC BB is given by: 


$$
\Delta_{B B, i}=d n_{B B, i}-\overline{d n_{B B, i}}=d n_{B B, i}-\frac{\Delta L_{B B, i}(B)-a_{2}^{B C S} \bullet d n_{B B, i}^{2}-a_{0}^{B C S}}{\overline{b_{l, i}^{B B}}}
$$

Similarly for the scan $i+1$ :

$$
\Delta_{B B, i+1}=d n_{B B, i+1}-\overline{d n_{B B, i+1}}=d n_{B B, i+1}-\frac{\Delta L_{B B, i+1}(B)-a_{2}^{B C S} \bullet d n_{B B, i+1}^{2}-a_{0}^{B C S}}{\overline{b_{1, i+1}^{B B}}}
$$

The instantaneous correction to $\mathrm{dn}_{\mathrm{EV}}$ is given by adding the linearly interpolated amount, according to:

$$
d n_{E V, i}(t) \rightarrow d n_{E V, i}(t)+\frac{d n_{E V, i}(t)}{d n_{B B, i}}\left(\frac{\Delta_{B B, i+1}-\Delta_{B B, i}}{2.954} \cdot t+\Delta_{B B, i}\right)
$$

where the time, $t$, is measured from the center of the $i^{\text {th }}$ scan measurement of the OBC BB.

Generalizing Eqn. 3, the radiance difference attributed to the Earth view path and the space view path (after reflection by the scan mirror) is given by:

$$
\Delta L_{E V}=L_{E V V_{P} A T H}-L_{S V S_{-} P A T H}=r_{E V}^{s m} L_{E V}+\left(r_{S V}^{s m}-r_{E V}^{s m}\right) L\left(T_{s m}\right)
$$

and similarly from Eqn. 8:

$$
r_{E V}^{s m} \overline{L_{E V}}+\left(r_{S V}^{s m}-r_{E V}^{s m}\right) \overline{L\left(T_{s m}\right)}=a_{0}^{B C S}(B)+\overline{b_{1}^{B B}(B)} \cdot d n_{E V}+a_{2}^{B C S}(B) \bullet\left(d n_{E V}\right)^{2}
$$

where the overstrike bar over the radiance terms (not the $b_{1}$ term) on the left hand side of Eqn. 20 indicate the appropriate RSR averaging similar to Eqn. 5.

Solving Eqn. 20 for the band averaged radiance from the Earth view, before the scan mirror reflection, representing the desired "at aperture" radiance, $\overline{\mathrm{L}_{\mathrm{EV}}(\mathrm{B})}$ yields:

$$
\begin{aligned}
\overline{L_{E V}(B)}= & \frac{l}{r_{E V}^{s m}(l)}\left[a_{0}^{B C S}(B)+\overline{b_{1}^{B B}(B)} \bullet d n_{E V}+a_{2}^{B C S}(B) \bullet\left(d n_{E V}\right)^{2}\right]- \\
& \frac{r_{S V}^{m}(l)-r_{E V}^{m}(l)}{r_{E V}^{s m}(l)} \cdot \frac{\int L\left(T_{T m}, l\right) \bullet R S R(B, l) \bullet d l}{\int R S R(B, l) \bullet d l}
\end{aligned}
$$


In summary $\overline{\mathrm{L}_{\mathrm{EV}}(\mathrm{B})}$ is determined by:

$$
\begin{gathered}
\overline{L_{E V}(B)}=L_{B V}\left[a_{0}^{B C S}(B), a_{2}^{B C S}(B), r_{S V}^{s m}, r_{B V}^{s m}, r_{B B}^{s m}, e_{c a v}(B), e_{B B}(B), D T_{B B}(B), R S R(B, l),\right. \\
\left.T_{B B}, T_{s m}, T_{C A V}, T_{i n s t} D N_{E V}, D N_{B B}, D N_{S V}\right]
\end{gathered}
$$

where the coefficients $\mathrm{a}_{0}{ }^{\mathrm{BCS}}(\mathrm{B}), \mathrm{a}_{2}^{\mathrm{BCS}}(\mathrm{B}), \rho_{\mathrm{SV}}^{\mathrm{sm}}, \rho_{\mathrm{EV}}^{\mathrm{sm}}, \rho_{\mathrm{BB}}^{\mathrm{sm}}, \varepsilon_{\mathrm{cav}}(\mathrm{B}), \varepsilon_{\mathrm{BB}}(\mathrm{B}), \Delta \mathrm{T}_{\mathrm{BB}}(\mathrm{B}), \mathrm{RSR}(\mathrm{B}, \lambda)$ are determined from pre-launch calibration measurements $\left(\Delta \mathrm{T}_{\mathrm{BB}}(\mathrm{B})\right.$ term is added to $\mathrm{T}_{\mathrm{BB}}(\mathrm{B})$ from the $\mathrm{BCS}$ to $\mathrm{OBC}$ calibration transfer process) and the remaining coefficients $\mathrm{T}_{\mathrm{BB}}, \mathrm{T}_{\mathrm{sm}}, \mathrm{T}_{\mathrm{CAV}}, \mathrm{T}_{\mathrm{inst}}, \mathrm{DB}_{\mathrm{EV}}, \mathrm{DN}_{\mathrm{BB}}, \mathrm{DN}_{\mathrm{SV}}$ are determined from on-orbit telemetry.

\subsection{On-orbit maneuver for Scan Mirror}

The MODIS scan mirror rotation produces a variable response versus scan angle (RVS) due to the 10.5 to 65.5 degree angle-of-incidence (AOI) variation across the 110 degree scan angle range, as a result of the different reflectance for $s$ and $p$ polarized light. In addition, the variable reflectance translates into a variable emission component. A systemlevel analytic expression of the variable RVS is complex and expected to vary with molecular contamination levels on-orbit. Attempts to measure the system-level RVS in the ambient environment were limited by the generally high instrument photon background and the need to operate with a mechanical cooler, which lead to additional instrument noise.

To meet the MODIS radiometric uncertainty requirements, it is planned to periodically measure the response vs scan angle on-orbit. The MODIS Science Team has requested that the AM spacecraft be oriented to scan deep space for up to about 30 minutes when the spacecraft is in the Earth shadow. This maneuver will provide the MODIS with a view above the horizon to observe the 3 Kelvin space background. During this orientation, the MODIS will respond to the scan angle dependent emission from the scan mirror. The scan angle-dependent emission can then be directly related to the scan angle-dependent reflectance by Kirchhoff's law. Model estimates of the scan mirror emission signal indicate adequate Signal to Noise Ratio for the LWIR bands. The GOES- 8 and -9 experiences demonstrate that these measurements can be performed to about 1 percent or less uncertainty for all emissive infrared bands. The GOES imager calibration for RVS is changed quarterly, Weinreb [10]. Consequently, the cold space scan maneuvers be implemented about yearly. 


\subsection{L1B Data Product Description}

The MODIS Level 1B software data product contains the radiometricall: fully calibrated instrument data in physical units at the original i] temporal resolution.

These data are broken into granules approximately five-minute long an Hierarchical Data Format (HDF), separated into the following four fi]

MODIS Level 1B 250 M Earth View Data Product which contains Eart observations in scientific units for the MODIS Bands 1 and meter spatial resolution;

MODIS Level 1B 500 M Earth View Data Product which contains Eart observations in scientific units from the MODIS Bands 1 anc at 500 meter spatial resolution, plus the Earth view obser MODIS Bands 3 through 7, at 500 meter spatial resolution;

MODIS Level 1B $1 \mathrm{KM}$ Earth View Data Product which contains Earth observations in scientific units from the MODIS Bands 1 th: aggregated at 1 kilometer spatial resolution, plus the Eart observations from MODIS Bands 8 through 36, at 1 kilometer resolution;

MODIS Level 1B OBC/E Product which contains on Board Calibrator observations in raw DNs from all MODIS bands, at their oric resolution, plus the Engineering data in engineering units. 
The aggregation algorithm used in the $500 \mathrm{~m}$ and 1,000 $\mathrm{m}$ Earth View fi: at [13].

Figure 4 shows the different components of the Level 1B HDF files. contains five types of metadata which describe the data components, their attributes. These are Core Metadata, Archive Metadata, Product Metadata, and Scientific Data Sets (SDS) Metadata. The Core, Archive metadata are stored once in each file as HDF global attributes. The 5 stored for each complete scan (one side of the scan mirror), are in 1 attributes for HDF-EOS required swath metadata, and HDF Vdata for Ler swath metadata. Vdata are tables of fixed field length, as in a vecto metadata are stored as Scientific Data set (SDS) attributes and do nc the Figure. The various types of metadata are used for different pur production and archive environment. Some are stored in a searchable $c$ tracking and queries by science users. The remainder serve as easily and summary information.

The science data in the Earth View files are instrument data and geol multiple SDSs in HDF-EOS Swath format. A small subset of internal $\subseteq$ are stored in the MODIS 1 KM Earth View Data Product for convenience visualization. The idiosyncrasies of the way the science data are st. the self describing capabilities of $\mathrm{HDF}$.

The corrected raw counts, dn*, from the instrument are stored as 151 software L1B data product for the reflected solar bands. The radiar product for each band in the reflected solar bands is computed as

$$
L_{B, D}=g_{0_{B, D}}+g_{B, D} \cdot d n *
$$

A linear calibration equation is used $\operatorname{fog}_{B, b}$ theserbapdadsatotte

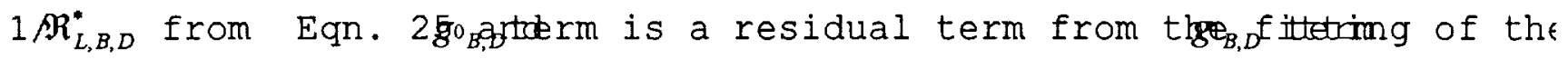


for the calibration data sets and corresponds with $L^{0}$. The g coeffic the granule-level metadata.

A similar approach to Eqn. 34 is used for the thermadn einsi sstianed bands, as the 15 bit integer. is ameinteger which produces the radiance for

$$
L_{B}=g_{0_{B}}+g_{B} \bullet \hat{d n} \quad(35) \text {. }
$$

In Eqn $3 \hat{b} \hat{n}$, is not reladued torkda* cannot be used for the thermal emiss: bands because the calibration for Eqn. 22 is not $g_{B}$ ling calibration terms are scaling terms for the thermal emissive bands, and are com granule and provided in the granule-level metadata. The calibration thermal emissive bands are embedded in the $g$ coefficients in the met is chosen to conserve output file space.

Invalid data fields are identified by having the highest order bit $s \in$ field is marked as invalid for the following reasons:

\author{
it was flagged as missing from the Level $1 \mathrm{~A}$ dataset; \\ the detector is dead; \\ the value was saturated; \\ there was a calibration failure;
}

Thus any data value larger than 32767 should be interpreted as invalj data fields that are flagged as missing from the Level $1 \mathrm{~A}$ dataset are regarded as unsigned 16 bit integers. For invalid data not flagged as $1 \mathrm{~A}$ dataset, the actual values stored in the file are the corrected $\mathrm{r}$ : the algorithm, stored as 15 bit unsigned integers, with the high ordt set to 1 . 
Associated with each instrument data value is uncertainty information Uncertainty information is reported as an index to conserve output $f$ Uncertainty Index is carried as a multiplicative factor to be applier spectral radiance specifications provided below. The index translate: value by use of the formula

$$
0.5 * \exp (\text { Uncertainty Index } / \mathrm{N})=\text { +Uncertainty Rang®3Mltiplier } \mathrm{T}
$$

The uncertainty is identified as a one-sigma uncertainty. The specifj for any band is obtained as the product of (the Uncertainty Range Mu specification uncertainty). The value of $\mathrm{N}$ for the thermal emissir value of $\mathrm{N}$ for the reflected solar bands is 9. . An Uncertainty Inde that the uncertainty has not been computed.. An Uncertainty Index $v$ in Bands 5, 6 or 7 indicates that the data for this pixel is contamir non-linearity and is not properly calibrated.

The specification for uncertainty in the reflected solar bands for th product tis: for all bands, all detectors. The specification in the for the radiance ptochlotfoirsall bands, all detectors. The specifica

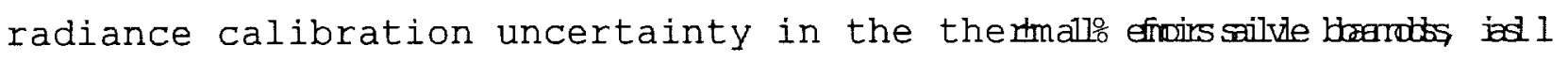
detectors, except for Band $\pm 2 \omega$. Whbichands Bands 31 and 324 whing. is

\subsection{Concluding Comments and Summary}

The material describing the at-launch MODIS radiometric product has been presented without a discussion of the actual polarization in the reflected solar bands. The specification for these bands is that the residual polarization sensitivity is not greater than 0.02 for bands with a center wavelength between 0.43 and $2.2 \mu \mathrm{m}$ for the Principal Scan Angles between $+45^{\circ}$ and $-45^{\circ}$. This performance specification is satisfied for most 
instances. Nevertheless, variations among detectors in some bands may impact the flatfielding determination described in Eqn. 23. Data from Bands $1-19$ are available only when the sensor is operated in the "Day Mode." Band 26 data is available in both Day Mode and Night Mode operations.

The MODIS principle radiometric products in the emissive infrared bands are radiance. The MODIS principle radiometric products in the reflected solar bands are radiance and reflectance factor. In all three instances an at-launch calibration equation is summarized. The traceability of the calibration standard is presented. The techniques to verify the atlaunch calibration throughout the mission lifetime are presented. The MODIS data user is encouraged to use the MCST Homepage on the World Wide Web for current calibration and characterization parameters. 


\subsection{References}

[1] William L. Barnes, Thomas S. Pagano and Vincent V. Salomonson, "Pre-launch performance of the MODerate Resolution Imaging Spectroradiometer (MODIS) on EOS AM-1," Transactions on Geoscience and Remote Sensing, this issue, 1998.

[2] Wayne E. Esaias, Mark R. Abbott, Otis W. Brown, Janet W. Campbell, Kendall L. Carder, Dennis K. Clark, Robert L. Evans, Frank E. Hoge, Howard R. Gordon, William P. Balch, Richard Letelier, Peter Minnett, "An Overview of MODIS Capabilities for Ocean Science Observations," Transactions on Geoscience and Remote Sensing, this issue, 1998.

[3] Christopher Justice, Dorothy Hall, Alfredo Huete, Ranga Myneni, Jan-Peter Muller, Steven Running, Alan Strahler, John Townshend, Eric Vermote and Zhengming Wan "The MODerate Imaging Spectrometer (MODIS) Mission to Planet Earth Enhanced Land Products Description", Transactions on Geoscience and Remote Sensing, this issue, 1998.

[4] Labsphere Corporation "A guide to reflectance materials and coatings," North Sutton, NH 03260, p. 3, 1996.

[5] J. Young, "PFM MWIR/LWIR radiometric calibration I: theory and measurement equations," SBRS Memorandum PL3095-N06555, Santa Barbara Remote Sensing, Goleta, CA, 1997

[6] D. Knowles, M. Jones, H. Montgomery, and L.L. Goldberg, "Algorithm Theoretical Basis Document 1996 Thermal Calibration Algorithm," MCST Reference No. G031, NASA's Goddard Space Flight Center, Mail Code 920.1, Greenbelt, MD 20771, pp. 48, 1996.

[7] G. Godden, S. Qiu and X. Xiong, "The MODIS Polarized Radiance Calibration Algorithm," MCST Memorandum, April, 1998

[8] M. Cafferty, Private Communication, 1995. 
[9] M. E. MacDonald "Testing of mirror as a function of wavelength and polarization at varying angles of incidence. Massachusetts Institute of Technology's Lincoln Laboratory, Lexington, MA, September 26, 1997.

[10] Michael Weinreb, Michael Jamieson, Nancy Fulton, Yen Chen, Joy Xie Johnson, Carl Smith, James Bremmer and Jeanette Baucom, "Operational calibration of the imagers and sounders on the GOES-8 and-9 satellites", NOAA Technical Memorandum NESDIS 44, Washington, DC, February 1997

[11] James J. Butler, "The Calibration Strategy for the Earth Observing System AM-1 Platform," Transactions on Geoscience and Remote Sensing, this issue, 1998.

[12] G.Thuillier, M. Herse, P.C. Simon, D. Labs, H. Mandel and D. Gillotay "Observations of the solar spectral irradiance from 200 to $850 \mathrm{~nm}$ during the ATLAS missions by the SOLSPEC Spectrometer," presented at New Developments and Applications in Optical Radiometry (NEWRAD '97), U. Arizona, Optical Sciences Center, Tucson, AZ, 85721, 27-29 October 1997.

[13] B. Guenther, "Ground calibration and in-orbit use of diffusers for Earth observations using MODIS as an example," presented at New Developments and Applications in Optical Radiometry (NEWRAD '97), U. Arizona, Optical Sciences Center, Tucson, AZ, 27-29 October 1997.

[14] Mash Nishihama, Robert Wolfe, David Salomonson, Frederick Patt, Jeffrey Blanchette, Edward Masuoka and Albert Fleig, "MODIS Level 1A Earth location; Algroithm Theoretical Basis Document, Version 3.0, SDST Reference No. 092, NASA's Goddard Space Flight Center, Mail Code 922, Greenbelt, MD 20771, 1997 


\subsection{List of Figure Captions}

Figure 1. Schematic of MODIS demonstrating the conversion of incident light to digital data output, demonstrating the relative location of the various targets which the sensor scan mirror observes, the fundamental optics and detectors layout and the digitizing electronics.

Figure 2a. The location in terms of the Principal Scan Angles within a MODIS scan, of various targets, including both the in-laboratory calibration sources, the on-board sensor calibrators and the Earth scenes. The Principal Scan Angle corresponds to the degrees of rotation the mirror moves between the center location for each target, with $0^{\circ}$ rotation selected at NADIR for the Earth scene.

Figure $2 \mathrm{~b}$. The angles of incidence (AOI) on the scan mirror within a MODIS scan of various targets is depicted.

Figure 3. Manufacturer specifications of the reflectance of a Spectralon ${ }^{\mathrm{TM}}$ diffuse scatter target is shown for the wavelengths between 0.25 and $2.5 \mu \mathrm{m}$. The MODIS Bands have center wavelengths between 0.42 and $2.10 \mu \mathrm{m}$. The MODIS Band 7 center wavelength nearly corresponds to the dip in the reflectance of Spectralon ${ }^{\mathrm{TM}}$ diffuser.

Figure 4. The MODIS Level IB HFD Format is depicted. The five types of metadata are identified, and the types of Vdata and Science Data Sets (SDSs) files are identified too. 


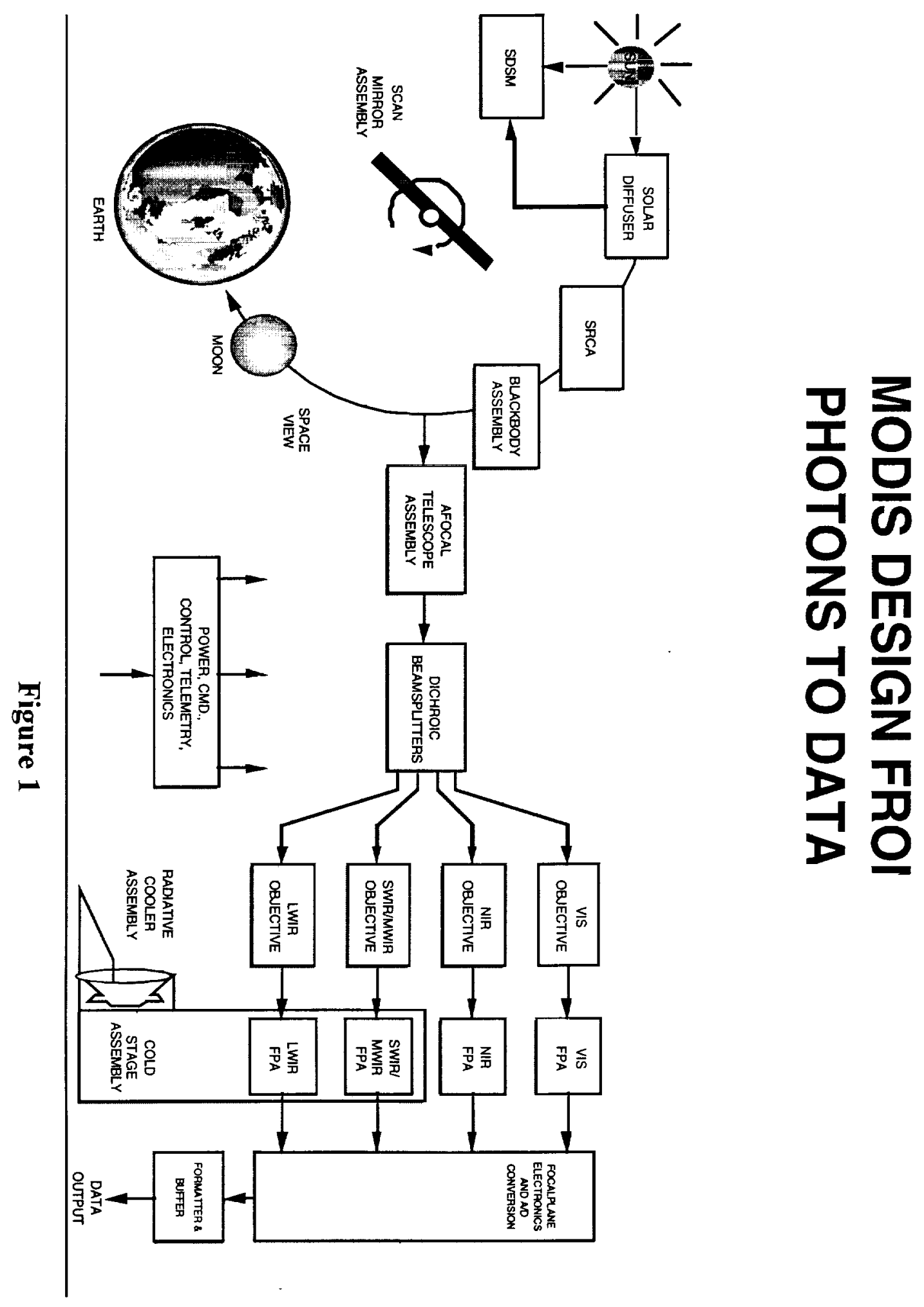



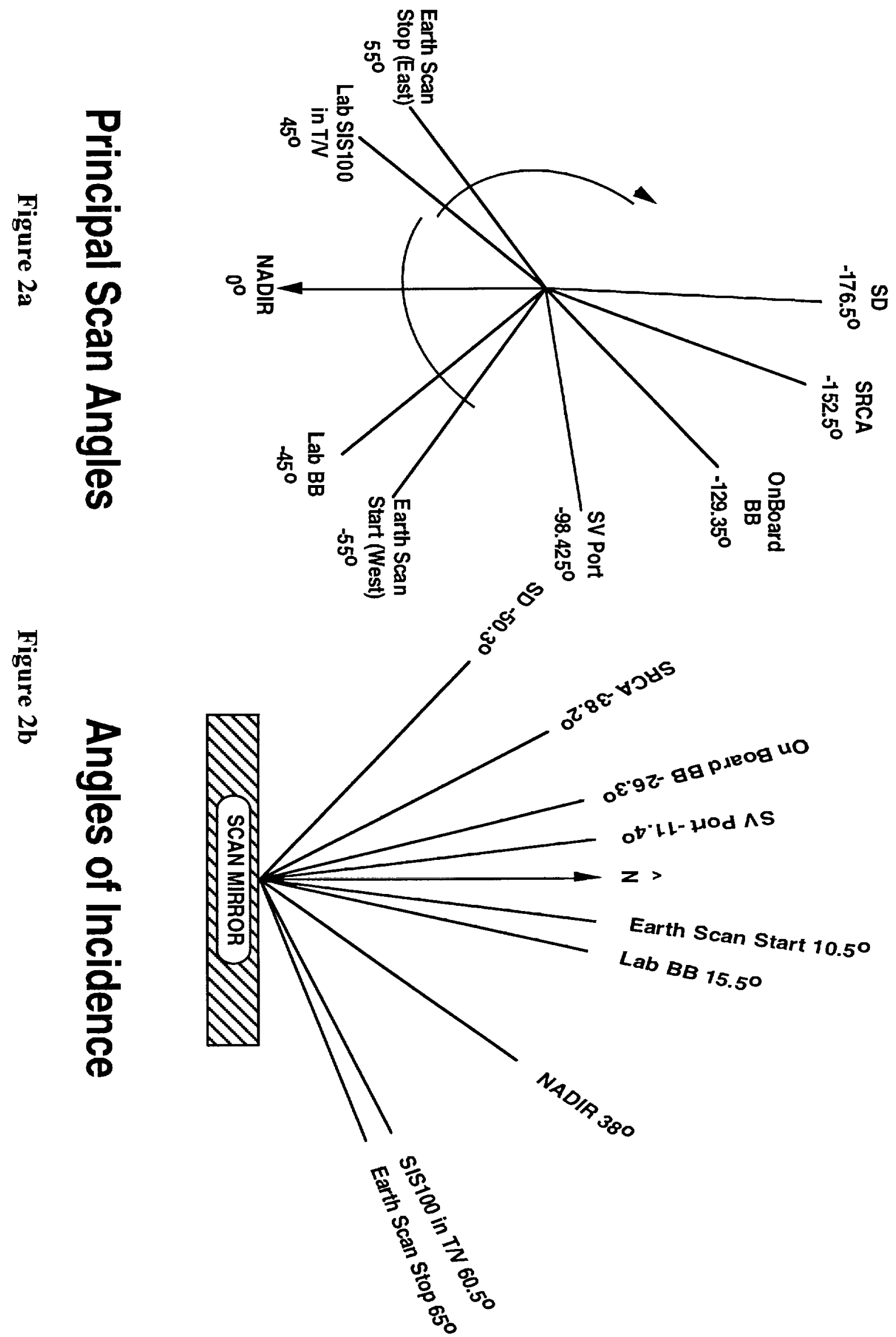


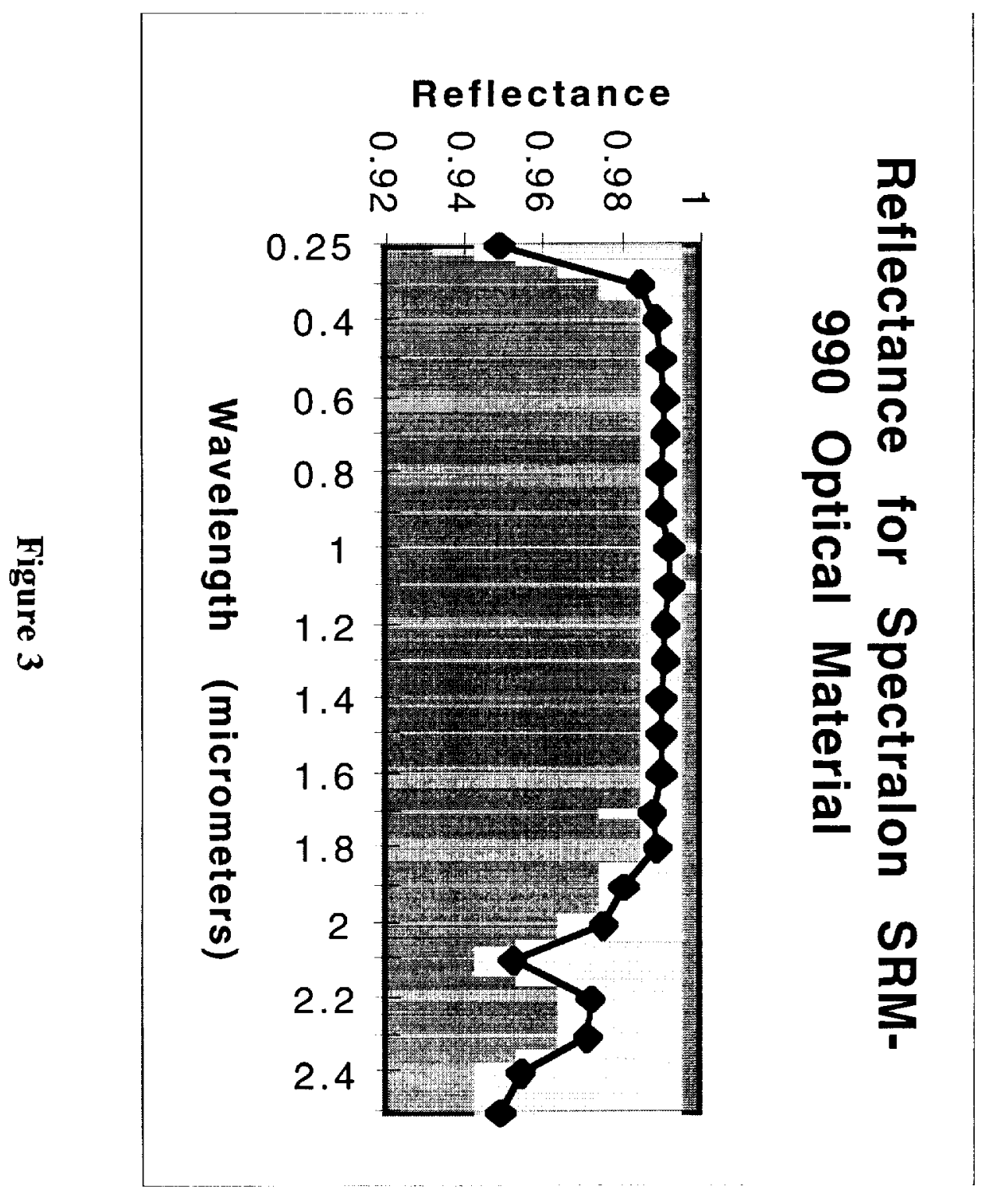


氮

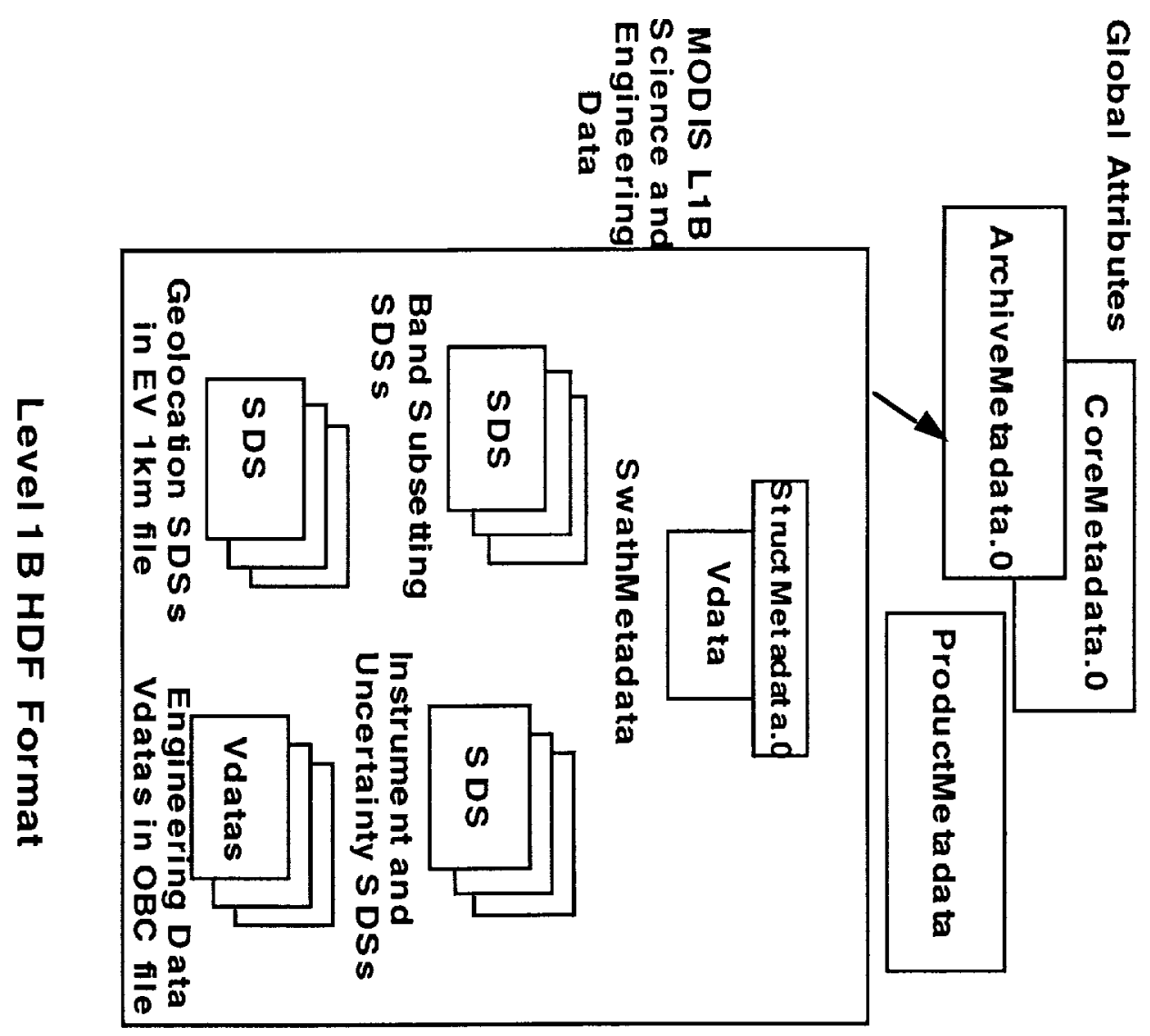

